\title{
YASMIN: petite pilule - grands effets
}

\section{Gert Printzen}

Dr, membre du Comité central de la FMH, responsable du département eHealth - Informatique et documentation médicales / Produits thérapeutiques

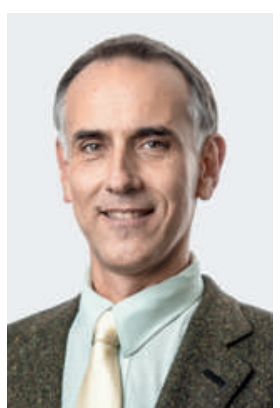

En 2008, une adolescente âgée de 16 ans qui prenait la pilule contraceptive "Yasmin» depuis environ 2 mois a dû être hospitalisée d'urgence suite à une perte de connaissance. Victime d'une grave lésion cérébrale sur embolie pulmonaire, la jeune fille est aujourd'hui lourdement handicapée.

Aussi bien le tribunal de district que le tribunal cantonal de Zurich ont minimisé la responsabilité du fabricant. Le 5 janvier 2015, le Tribunal fédéral est parvenu à la même conclusion: l'information donnée à la patiente sur la pilule avait été correcte, le fabricant a fourni des indications suffisantes sur les risques de

\section{Il est essentiel d'informer les patientes} de manière complète et compréhensible des possibles risques, notamment pour les médicaments soumis à ordonnance.

la pilule Yasmin dans la notice d'information destinée aux patientes. Pour tous les médicaments soumis à ordonnance, la responsabilité d'informer revient en premier lieu au médecin traitant.

Pour de nombreux médicaments, et en particulier ceux prescrits par ordonnance, les patients ne disposent pas toujours des connaissances spécifiques nécessaires pour évaluer les bénéfices et les risques du produit. Plus que toute autre, la question de la contraception orale revêt une importance cruciale pour la pratique gynécologique. Lors d'une première prescription, la pratique habituelle consiste à disposer de suffisamment de temps pour informer la patiente de tous les risques ou effets secondaires liés à la pilule, mais également pour lui montrer le chemin pour une meilleure utilisation et satisfaction avec la contraception orale prescrite.

En 2013, le Comité pour l'évaluation des risques en matière de pharmacovigilance (PRAC) de l'Agence européenne des médicaments (EMA) a pour la première fois réalisé une évaluation des pilules contraceptives sous l'angle de la nouvelle réglementation sur la pharmacovigilance: en novembre 2013, le Comité des médicaments à usage humain (CHMP) de l'EMA est arrivé à la conclusion que les bénéfices des contraceptifs hormonaux combinés ( $\mathrm{CHC}$ ) pour prévenir les grossesses non désirées restaient supérieurs au risque de thromboembolies veineuses (VTE) et que ce risque avéré était faible pour tous les $\mathrm{CHC}$. Cette analyse a également mis en évidence l'importance d'une information claire et actuelle des femmes qui utilisent ces médicaments, mais également du personnel médical spécialisé qui les conseille et les prend en charge. En conséquence, les notices d'information des $\mathrm{CHC}$ ont été actualisées.

Compte tenu du risque reconnu de thrombose, l'Allemagne a décidé que toutes les informations d'utilisation ou pour spécialistes des contraceptifs oraux combinés devaient être complétées à partir du mois d'août 2014. Ce faisant, l'Institut fédéral pour les médicaments et les produits médicaux (BfArM) a appliqué la décision européenne du 16 janvier 2014.

Il est essentiel et indiscutable que les femmes doivent être informées du risque de VTE, de ses signes et de ses symptômes, et que les médecins doivent prendre en compte les facteurs de risques individuels au moment de prescrire un contraceptif. Mais finalement, les patientes sont seules à décider si elles souhaitent prendre la pilule ou choisir un autre moyen de contraception voire y renoncer. Pour cette raison, la

\section{Une amélioration possible: de nouvelles mises} en garde concernant les thromboses.

FMH ne comprend pas que les informations contenues dans la notice d'emballage ne soient pas juridiquement pertinentes. Aux Etats-Unis, le fabricant avait dû renforcer les avertissements sur les emballages de ses pilules suite au dépôt de plusieurs centaines de plaintes. Il en va tout autrement en Suisse. $\mathrm{Au}$ final, les analyses «bénéfices/risques» et les taux d'incidence restent des statistiques qui, s'ils permettent de mettre en évidence l'existence de cas isolés, ne peuvent malheureusement ni les prévenir ni les appréhender dans leur dimension tragique individuelle. 